\title{
Quand le patrimoine alimentaire innove
}

Analyse sociologique des processus d'innovation patrimoniale alimentaire au service des territoires

Jacinthe Bessière

\section{(2) OpenEdition}

1 Journals

Édition électronique

URL : http://journals.openedition.org/tourisme/182

DOI : 10.4000/tourisme.182

ISSN : 2492-7503

Éditeur

Éditions touristiques européennes

\section{Édition imprimée}

Date de publication : 1 juin 2013

Pagination : $37-51$

ISSN : 2109-5671

\section{Référence électronique}

Jacinthe Bessière, "Quand le patrimoine alimentaire innove », Mondes du Tourisme [En ligne], 7 | 2013 mis en ligne le 30 septembre 2015, consulté le 19 avril 2019. URL : http://journals.openedition.org/ tourisme/182 ; DOI : 10.4000/tourisme.182

\section{c)}

Mondes du tourisme est mis à disposition selon les termes de la licence Creative Commons Attribution - Pas d'Utilisation Commerciale - Pas de Modification 4.0 International. 


\title{
Quand le patrimoine alimentaire innove
}

\section{Analyse sociologique des processus d'innovation patrimoniale alimentaire au service des territoires}

\author{
JACINTHE BESSIÈRE \\ [bessiere@univ-tlse2.fr] \\ Maitre de conférences en sociologie \\ Centre d'étude et de recherche Travail, Organisation, Pouvoir (Certop) - \\ CNRS - UMR 5044, Institut supérieur du tourisme, de l'hôtellerie et de \\ I'alimentation (Isthia), Université de Toulouse - Le Mirail
}

Résumé. La production agricole sous-tend aujourd'hui une dynamique de patrimonialisation marquée par une valorisation tant économique que socioculturelle de ses ressources. Accompagnant les stratégies de développement des territoires ruraux, et répondant à une demande sociale croissante, ces processus de patrimonialisation renouvellent la vocation agricole des espaces ruraux qui remplissent désormais de nouvelles fonctions, culturelles, touristiques, sociales, etc. Cette contribution, qui s'appuie sur un programme de recherche commandité et soutenu par le conseil régional de Midi-Pyrénées, a pour objectif d'analyser, à travers le prisme de l'innovation, les processus de valorisation des patrimoines alimentaires, entendus comme un ensemble d'éléments matériels ou immatériels liés à des productions et à l'univers agricole ainsi qu'à un héritage collectif territorial. Nous émettons l'hypothèse que les dynamiques de construction du patrimoine consistent à actualiser, à adapter, à réinterpréter des traits puisés dans l'histoire d'un groupe (savoirs, savoir-faire), c'est-à-dire à combiner héritage et innovation. À partir de l'analyse de trois territoires ruraux midipyrénéens, il s'agit de comprendre les mécanismes de patrimonialisation alimentaire (construction, sauvegarde, évolution) à travers la composante "innovatrice" ou "créatrice".

Abstract. Nowadays, agricultural production underpins a heritigization dynamic marked by an economic, but also sociocultural promotion of its resources. Accompanying the development strategies of rural territories, and satisfying a growing social demand, these heritigization processes are renewing the agricultural vocation of social spaces which now fulfill new cultural, touristic, social functions, etc. The present article, which draws on a research program sponsored and promoted by the Conseil régional of Midi-Pyrénées, strives to analyze, through the prism of innovation, the processes promoting food heritage, understood as a series of tangible or intangible elements linked to productions and to the agricultural field, as well as territorial collective heritage. We can hypothesize that the construction dynamics of heritage consist in updating, adapting, reinterpreting characteristics drawing on the history of a group (knowledge, know-how), i.e. combining heritage and innovation. The analysis of three Midi-Pyrénées rural territories will allow us to understand the mechanisms of food heritigization (construction, conservation, evolution) through the "innovative" or "creative" component. 
$\mathbf{L}$ e secteur agricole et une partie des productions agroalimentaires connaissent aujourd'hui un mouvement indéniable de patrimonialisation, traduisant des logiques identitaires et socioculturelles ancrées dans les territoires ruraux. Ces dernières, portées et revendiquées par une demande sociale croissante, ouvrent la voie à des dynamiques de productions et de valorisation patrimoniales qui accompagnent les stratégies de développement des territoires. Aussi, l'avènement de nouvelles pratiques et représentations alimentaires, tout comme l'engouement actuel pour les terroirs, réinterroge et renouvelle la vocation agricole des espaces ruraux donnant à voir de nouvelles fonctions culturelles, touristiques ou sociales. Cette contribution, qui s'appuie sur un programme de recherche commandité et soutenu par le conseil régional de Midi-Pyrénées, a pour objectif de comprendre les processus de valorisation des patrimoines alimentaires en espace rural, sous l'angle de l'innovation ${ }^{(1)}$. Nous définissons le patrimoine alimentaire comme l'ensemble des éléments matériels et immatériels constituant les cultures alimentaires et définis par la collectivité comme un héritage partagé : "Concrètement, il se compose de l'ensemble des produits agricoles, bruts et transformés, des savoirs et savoir-faire mobilisés pour les produire, ainsi que des techniques et objets culinaires liés à leur transformation. Ce patrimoine comprend également les savoirs et pratiques liés à la consommation (manières de table, formes de sociabilité, symbolique des aliments, objets de la table...) et à la distribution alimentaire (marchés de pays, vente à la ferme)" (Bessière et Tibère, 20I I). S'il constitue une grille de lecture, une voie de compréhension des sociétés en quête d'identité, le patrimoine alimentaire est également aujourd'hui le lieu de projets et d'actions de mobilisation, s'inscrivant dans des dynamiques d'innovation territoriale, notamment touristique (Bessière, 2013). Il constitue un levier de développement local pour les territoires ruraux ouvrant la voie à de nouvelles formes de diversification socio-économique. Empreinte du temps, héritage transmis ou à transmettre, le patrimoine s'inscrit dans des logiques de perpétuation et de reproduction. Néanmoins, au-delà de cette continuité, le patrimoine se révèle espace d'invention, de recomposition et d'innovation propulsant le passé dans l'avenir. Entre héritage et innovation, entre histoire et avenir, les patrimoines se reconfigurent, et se positionnent comme des construits sociaux, des productions collectives issues des territoires et des groupes. C'est sur ces dynamiques d'innovation et de recomposition que nous nous proposons de bâtir notre réflexion. En quoi et comment l'innovation investit-elle le processus de patrimonialisation, ici alimentaire ? En quoi les stratégies de valorisation patrimoniale intègrent-elles l'innovation sans pour autant inhiber l'ancrage spatial et temporel des patrimoines alimentaires ?

L'innovation, que nous analysons dans cet article comme un processus social, peut prendre appui sur le patrimoine actif, ou réhabiliter le patrimoine dormant ; elle peut enfin s'appuyer sur un patrimoine construit, inventé (Poulain, 1997). Ainsi, les cultures alimentaires sont en construction permanente. Elles sont mobiles, voire instables, et font l'objet de différentes formes d'innovation : innovation de produit, technologique, commerciale ou marketing, innovation partenariale, organisationnelle, stratégique ou territoriale. Constituée d'acteurs, de stratégies, d'usages et de conflits, l'innovation, appliquée à la valorisation des patrimoines alimentaires, traduit un processus complexe dont nous tenterons l'analyse dans cet article. En outre, ce dernier interroge l'avènement et la composition des processus d'innovation patrimoniale s'inscrivant dans des logiques de développement local pour les territoires. Il met en avant les processus de diversification et d'enrichissement des activités économiques et sociales d'un territoire à partir de l'innovation patrimoniale alimentaire.

Les résultats présentés s'appuient sur l'analyse de trois territoires ruraux midi-pyrénéens, choisis en raison de leur engagement dans les stratégies d'innovation $^{(2)}$ : le groupe d'action locale (Gal) Aubrac, Olt et Causses, dans le Nord-Aveyron, le Gal Association pour la modernisation et l'innovation économique AMIE, dans le Sud-Aveyron, le Gal Pays d'Armagnac, dans le Gers. La démarche méthodologique choisie est de nature qualitative, visant la compréhension approfondie des 
mécanismes, des stratégies d'innovation et de patrimonialisation à l'œuvre sur les territoires ${ }^{(3)}$.

Dans une première partie, nous analyserons les principaux facteurs qui influent sur le processus de construction de l'innovation, en questionnant les sources de l'invention patrimoniale alimentaire puis les leviers de l'appropriation de nouvelles pratiques sociales. En quoi et comment une invention, ici spécifiquement associée à l'univers des patrimoines alimentaires, se diffuse, se propage, s'accepte comme une innovation? D'où partent les innovations? Quels chemins empruntentelles ? Puis, dans une seconde partie, nous tenterons d'expliciter l'articulation entre innovation et patrimonialisation alimentaire, en analysant le rapport à la tradition en tant qu'élément influant sur les stratégies observées. Si l'identification du patrimoine recouvre le plus souvent une dimension unitaire ou unificatrice, elle peut aussi être source de division et de désunion sociale. Le patrimoine alimentaire et l'innovation qui y est associée apparaissent comme un enjeu de représentations contradictoires, engendrant des relations parfois conflictuelles entre les acteurs.

\section{LES CHEMINS \\ DE L'INNOVATION : \\ FONDEMENTS ET SOURCES DE \\ L'INNOVATION PATRIMONIALE \\ ALIMENTAIRE}

Si nous reprenons les analyses de Norbert Alter, l'innovation décrit le processus par lequel une invention s'insère dans un système social.

L'innovation se construit alors à travers le changement de pratiques et de représentations sociales, indiquant par là même la diffusion et l'assimilation du processus innovant. "Celle-ci [l'invention] représente une nouvelle donne, la création d'une nouveauté technique ou organisationnelle, concernant des biens, des services ou des dispositifs, alors que l'innovation représente l'ensemble $d u$ processus social et économique amenant l'invention à être finalement utilisée ou pas" (Alter, 2002). Cette distinction entre invention et innovation décrite précisément par Alter nous amène à considérer l'invention ou la création en position d'antériorité par rapport à l'innovation. L'innovation se construit dans la diffusion et l'assimilation de l'invention, à travers le changement induit de pratiques et de représentations sociales (Alter, 2000). Cependant, ce passage de l'invention à l'innovation semble guidé dans le cas des patrimoines alimentaires par un ensemble de facteurs et de déterminants qui varient selon les contextes sociaux et territoriaux.

\section{Rupture, mobilités et esprit de création}

En premier lieu, c'est une volonté de rupture qui s'avère source d'invention patrimoniale. Sorte de fracture dans un parcours professionnel ou personnel, l'invention dans le champ des patrimoines alimentaires peut résulter d'une situation de difficulté, d'échec, de fragilité ou de peur.
L'obtention de labels ou l'intégration de démarches qualité constituent, par exemple, des formes d'invention dont les fonctions sont de répondre à la crise alimentaire mais aussi de compenser un déficit ou une économie fragile. La structuration des acteurs autour d'une filière ou d'un signe de qualité (appellation d'origine protégée, indication géographique protégée, label rouge) relève le plus souvent d'une action défensive visant le sauvetage ou la relance d'une production tombée en désuétude. À titre d'exemple, la crise alimentaire de la vache folle a provoqué ou a consolidé une organisation des acteurs autour des filières de qualité et a donné naissance à une re-valorisation de certains produits "viande" ou "charcuterie". "Après la crise de la vache folle, on a dî se spécialiser dans la race Aubrac. Et on s'est replié sur des réseaux de commercialisation en quête de traçabilité et de qualité. On est arrivé au bon moment; en fait, notre succès est parti de la vache folle. Ça a été le déclencheur" (charcutier, Gal Aubrac, Olt et Causses, Nord-Aveyron).

La démarche d'innovation se construit également dans le désir de différenciation, par opposition à un système jugé dominant et oppressant. L'objectif est ici de rompre au moins en partie avec les règles et normes établies. Les nombreuses initiatives innovantes de valorisation, avec notamment le lancement de nouveaux produits, observées dans les territoires du Gal AMIE SudAveyron (fabrication d'apéritifs 
régionaux, de miel, de yaourts, etc.) et du Pays d'Armagnac, dans le Gers (relance de la moutarde, innovations autour du vin, etc.), se caractérisent par cette volonté de s'écarter du modèle économique en place marqué par l'hégémonie de quelques filières traditionnellement implantées sur les territoires. Ces dernières, à l'instar de la filière roquefort dans le SudAveyron, de la filière armagnac et foie gras dans le Gers, ont donné naissance à des formes alternatives de développement fondées sur l'innovation. "Plus les choses allaient et moins je me trouvais à l'aise dans mon travail, c'est comme si j'allais à l'usine, entre le travail et ce que j'avais dans la tête, je croyais faire l'inverse de ce que je pensais. [...] Petit à petit, je me suis sentie mal à l'aise, je suis partie du Gaec en 2000 et je me suis lancée dans la fabrication de yaourts" (productrice de yaourts, Gal AMIE, Sud-Aveyron).

En second lieu, les phénomènes de mobilités s'avèrent source d'innovation dans le champ des patrimoines alimentaires. Qu'ils soient professionnels, touristiques ou de loisirs, les mouvements circulatoires irriguent nos trois territoires d'enquête, impulsant changement et renouvellement dans les stratégies de valorisation patrimoniale.

En effet, les touristes étrangers ou français, les néo-ruraux ou les résidents secondaires induisent, par un regard neuf et extérieur, de nouvelles représentations et pratiques sociales chez les populations locales, jusqu'à intervenir directement dans les projets de valorisation et les formes d'innovation. Ici, "l'extérieur", "l'inconnu" est source d'inspiration, de créativité. L'apport de la nouveauté ou la différenciation se construit dans la confrontation, dans la comparabilité sociale, territoriale ou encore à la marge (Alter, 2000). Les clientèles touristiques jouent indéniablement un rôle de reconnaissance des produits et des ressources à l'échelle locale ; elles apportent, par leurs pratiques, leurs usages et leurs représentations du patrimoine alimentaire, un gage de reconnaissance et de garantie extérieure. Elles sont également un levier de réappropriation locale, en réactivant ou en impulsant un nouveau regard sur le patrimoine alimentaire, parfois indifféremment laissé à l'écart. Le phénomène touristique contribue ainsi à la sensibilisation et à l'appropriation des patrimoines alimentaires locaux. Par effet de confrontation et de différenciation, le regard touristique concourt à la prise de conscience et à la reconnaissance des marqueurs culturels, et intervient dans le renouvellement ou la valorisation des offres alimentaires locales. "Le réseau 'Visite de ferme', il est né parce qu'il y a eu un désir des touristes, parce que quand ils arrivaient sur Roquefort, ils ne voyaient pas les brebis dehors, il y avait des fromages factices dans les caves, et c'est vrai que c'est assez désagréable" (agricultrice, réseau "De ferme en ferme", Gal AMIE, Sud-Aveyron).

Les mécanismes de métissage, ici alimentaire (Corbeau et Poulain, 2002), à la croisée d'héritages et d'emprunts, sont le résultat d'alliances ou de mariages nés des phénomènes de mobilités. L'analyse montre une récurrence des discours sur l'influence des réseaux et des partenariats, notamment extérieurs au territoire. Voyages d'études, déplacements, observations extérieures, suivi de formations, apports de connaissances ou curiosité sont autant de vecteurs d'innovation. Ces diverses mobilités (touristiques, résidentielles, professionnelles) se traduisent par l'introduction de nouvelles représentations du territoire, de nouveaux rapports à l'alimentation et aux cultures locales. Les migrants, de plus ou moins longue durée, par leurs tentatives de réinvention d'un "làbas" dans un nouvel "ici", peuvent mobiliser des processus de création, d'invention sur les patrimoines (Fourcade et Legrand, 2008). En Pays d'Armagnac, nous pouvons noter l'importation de produits "traditionnels" issus d'autres territoires. L'innovation peut résider ici dans l'action d'implanter ou d'importer un produit sur un territoire.

Ces influences migratoires agissent selon un référentiel temporel mais également spatial, comme de réels leviers à l'innovation: "Après, en termes d'innovation, on peut faire aussi des tagines de poulet, des amalgames, des mélanges en fonction des attentes du consommateur d'aujourd'bui" (traiteur, Gal Pays d'Armagnac, Gers).

Notons enfin le rôle du regard extérieur comme levier d'innovation, porté notamment par les "experts" ou "penseurs" des territoires, solli- 
cités à des fins de conseil, d'accompagnement ou de revitalisation territoriale. L'appui des cabinets d'études, consultants, chercheurs, enseignants du supérieur constitue un outil de déclenchement de l'innovation. En Aubrac, au sein du Gal AOC, nous pouvons rappeler le rôle joué par l'auscultation scientifique commanditée par le CNRS, réalisée sur le territoire dans les années 1970 ; la présence d'un regard objectivant, fruit de recherches conduites par divers chercheurs de disciplines différentes, a déclenché un sentiment de volontarisme, de solidarité et de mobilisation agricole autour des ressources agroalimentaires et patrimoniales. Comme un électrochoc, et dénonçant la perte d'un héritage, elle est à la source de stratégies d'innovations organisationnelles, technologiques autour des patrimoines alimentaires du territoire Aubrac. Elle a eu pour fonction de revitaliser le lien entre un milieu naturel et des hommes. La réactivation de cette liaison, ou de cette passerelle entre les hommes et leur milieu, constitue une innovation stratégique ou sociétale.

En dernier lieu, le processus d'invention patrimoniale doit également ses origines à l'esprit de création et à la conviction personnelle, facteurs incontournables de l'innovation. Dans les années 1970, dans le SudAveyron (Gal AMIE), nombreux sont les acteurs qui ont innové, dans un premier temps par conviction et stratégie personnelle, de manière à maintenir la vocation agricole de certaines terres vouées alors à dis- paraître dans un camp militaire. "On est ici sur le Larzac dans une ferme ou je me suis installé en 1975 et qui faisait l'objet de l'extension du camp militaire. Je m'y suis installé pour conserver la vocation agricole des lieux" (producteur d'apéritifs régionaux, Gal AMIE, SudAveyron). "Cela permet d'utiliser des terrains abandonnés pour qu'ils servent à quelque chose. C'est de créer une activité sur place" (productrice de safran, Gal AMIE, SudAveyron).

En outre, l'innovation est indéniablement marquée par "l'esprit" d'ouverture, l'échange et la circulation d'idées et de concepts. Nous pouvons nous demander finalement s'il existe une "culture de l'innovation", une capacité d'ouverture d'esprit, caractérisée par la volonté incessante d'inventer, de créer ou d'avancer. "L'esprit Bras" dans le Nord-Aveyron est par exemple fortement marqué par la création. La vision des chefs cuisiniers, Sébastien et Michel Bras, est innovante en ellemême, définissant le patrimoine alimentaire comme une construction, un condensé d'innovation permanente, qui s'invente chaque jour dans une assiette et dans le rapport aux choses. Le célèbre "gargouillou de jeunes légumes" n'est-il pas la preuve d'une imagination hors du commun, qui offre sur une même assiette une association de différents légumes, graines, bulbes et herbes champêtres, en partie issus du territoire et évoquant le printemps en Aubrac?

L'innovation est alors considérée comme un état d'esprit caractérisé par l'éveil de la curiosité qui mène à des formes de créativité. S'il est impératif de comprendre que l'innovation est en soi quelque chose de nouveau, nous devons par conséquent comprendre qu'elle se situe en dehors de nos habitudes, de nos cadres de référence et parfois audelà de notre imagination. Une partie des personnes interrogées nous ont amenée à considérer la nécessité de s'immerger dans des environnements différents de ceux que nous côtoyons quotidiennement, comme leviers à l'innovation. Une forme d'inspiration en quelque sorte, conduisant à confronter les individus à des habitudes, à des savoirs et savoir-faire différents de ceux qu'ils pratiquent au quotidien. Talent, ingéniosité ou curiosité semblent indispensables dans la production d'invention alimentaire et donc d'innovation.

Enfin, si l'inventeur peut être dénué de sens pratique, l'innovateur cherche un marché ou un usage pour ses découvertes ; diffusion souvent lente comme en témoignent les analyses des historiens. Si nous reprenons les analyses de Gérald Gaglio, de nombreux innovateurs rencontrés se définissent avant tout comme des inventeurs marqués par des "croyances", "croyances positives et normatives" (cf. Norbert Alter, cité dans Gaglio, 20। I) leur permettant d'affronter l'adversité et la résistance. Dotés d'une certaine force psychique de conviction et de persuasion, ils véhiculent auprès des autres de nouvelles façons de penser, d'agir et de faire. 


\section{Appropriation collective et acquisition de nouvelles pratiques sociales}

L'appropriation collective par l'acquisition de nouvelles pratiques autour du patrimoine valorisé constitue un deuxième temps du processus d'innovation. Fait d'une rencontre entre une nouveauté et un groupe social, ce second moment attribue la légitimité à la nouveauté, transformant l'invention en innovation. On peut donc essayer de questionner ce qui facilite la transformation en innovation, ce qui détermine cette co-construction d'usages collectifs autour de l'innovation patrimoniale alimentaire. Comment des comportements individuels souvent minoritaires transforment-ils des conduites collectives et construisent-ils de nouvelles règles, de nouvelles normes (Alter, 2000) ?

Dans le cas des patrimoines alimentaires, l'étude montre que cette co-construction s'appuie sur l'ancrage des productions alimentaires et donc sur leur dimension patrimoniale. Si l'on part du postulat que les patrimoines alimentaires se définissent par leur inscription territoriale (lien au lieu) et temporelle (lien au temps) (Bessière, 200I), nous pouvons en déduire, que l'innovation, loin de s'opposer à la tradition, demeure intimement liée à l'héritage alimentaire.

Nous retrouvons la présence de l'ancrage temporel et de la dimension traditionnelle des productions alimentaires locales comme intervenant dans la construction du processus d'innovation. Le passé semble dans de nombreux cas nourrir l'innovation et le projet. C'est le cas par exemple de phénomènes d'imitation et de copiage qui relèvent plus de pratiques antérieures que d'une réelle forme de création. "La moutarde ce n'est pas une invention, c'est un produit ressuscité ici ; les vieux trucs, c'est une vraie ressource" (agriculteur, producteur de moutarde, Pays d'Armagnac, Gers).

Quelques restaurateurs rencontrés centrent notamment leur discours sur la nécessité d'une innovation s'appuyant sur la tradition et l'héritage familial. "Mes parents et mes grands-parents étaient restaurateurs, c'est une tradition. Quand j'ai repris l'affaire de mes parents, j'ai repris leur manière de faire la cuisine et jai essayé d'aller plus loin, d'apporter quelque chose, de nouvelles façons de travailler le produit, sachant qu'il $y$ a des produits auxquels on ne peut pas enlever la cuisson comme on le faisait autrefois, des cuissons longues. Même si on veut moderniser, on ne peut pas enlever ça, il faut s'appuyer dessus" (restauratrice, Gal Aubrac, Olt et Causses, Aubrac).

L'ancrage temporel présenté ici contribue à la légitimité du processus d'innovation en favorisant son processus d'adoption et de diffusion. Le patrimoine sert de point d'appui, de levier à l'innovation et à d'autres démarches d'invention ou de création alimentaire. La tradition apparaît ainsi comme une ressource pour l'innovation, favorisant l'appropriation et la diffusion de nouveaux usages. "J'ai repris le laboratoire vers 1998 de la dame qui faisait les tripoux, les petites de Laguiole, un tripoux cousu main avec une bonne réputation et une bonne renommée. J'ai repris ce laboratoire avec une petite boutique devant. Puis j'ai fait à partir de là ma spécialité qui était le foie gras et tout ce qui est dérivé $d u$ canard" (responsable de conserverie, Gal Aubrac, Olt et Causses, Aubrac).

Autre condition favorable au processus d'innovation patrimoniale : l'ancrage spatial des productions patrimoniales. Ici, c'est le territoire, ou le terroir entendu comme support spatial et culturel de production de ressources spécifiques, qui favorise la diffusion et l'appropriation de nouvelles pratiques. L'innovation trouve son sens et son origine dans les caractéristiques géographiques et culturelles du lieu. La notion de terroir demeure ainsi largement évoquée dans les discours et se définit comme un lieu privilégié d'expérimentation et d'anticipation. Nous retrouvons les deux dimensions structurantes des terroirs (Bessière, 201 I) :

- la dimension géographique des productions agricoles, liée aux spécificités physiques du lieu. Elle décrit des productions de terroirs dont les caractères et les qualités sont issus d'une aptitude et de potentialités physiques particulières au milieu. L'innovation consiste ici à s'adapter ou à proposer des expériences ou des offres nouvelles calquées sur les attributs essentiellement naturels (sols, climat, etc.) du territoire ; - la dimension socio-historique des terroirs, qui renvoie à la qualification identitaire de la société locale. Lié à 
l'histoire et à la mémoire de la collectivité, doté de particularismes socioéconomiques locaux mais aussi d'un ensemble de pratiques et de représentations communes au lieu, le terroir traduit une appartenance à une même communauté. L'innovation consiste ici à prendre en compte la profondeur historique et culturelle des territoires, en puisant dans l'existence des savoir-faire transmis et accumulés par la communauté.

Chez Michel et Sébastien Bras, en Aubrac, l'innovation s'appuie sur le rapport au lieu, sur une certaine vision du terroir d'appartenance. Leur cuisine se veut être le miroir d'un lieu, d'un milieu s'inspirant des attributs naturels de l'Aubrac et s'inscrivant par là même dans une logique de développement territorial (Etcheverria, 20। I). L'ancrage du processus d'innovation est ici essentiellement spatial et géographique. "Dans tous les cas, ce qu'il y a dans mes assiettes, c'est tout mon état émotionnel, il n'y a pas de mystère... Par exemple, je fais un plat de terroir qui dérange beaucoup : la lotte avec de l'olive noire ; c'est le plateau de l'Aubrac, j'ai mis deux ans à vouloir retraduire cette image : plafond bas, ombres, trous dans les nuages... C'est ce rapport de l'ombre et de lumière que je voulais traduire dans une assiette. La lotte est claire, nacrée. Je la fais pocher dans une buile très noire, très profonde... C'est ceci le terroir! Mon gargouillou : étymologiquement, c'est un plat de cochon et de patates; moi, je l'ai retraduit avec la vision des choses; le gargouillou de Michel
Bras c'est le printemps sur l'Aubrac avec des fleurs, du mouvement... Je pourrais raconter tous mes plats de cette façon..." (Michel Bras, chef cuisinier, Gal Aubrac, Olt et Causses, Nord-Aveyron).

L'ancrage au temps et à l'espace influe directement sur les stratégies d'innovation. En son absence, nous observons des effets de résistance notables, empêchant l'appropriation et la diffusion collectives de l'invention. L'innovation sans fondements patrimoniaux, c'est-à-dire sans accroche spécifiquement spatiale et temporelle, semble être mal perçue et mal acceptée tant par la société locale que la population touristique. Olivier de Sardan (1995) pointe ce facteur comme déterminant dans la réussite et la diffusion des innovations.

La présence des réseaux est un autre facteur clé qui favorise l'appropriation de la nouveauté par le groupe social, permettant le plus souvent la naissance et l'institutionnalisation de l'innovation. Nous avons pu observer l'importance des réseaux informels qui se tissent dans l'interconnaissance, la proximité sociale et culturelle des individus, mais aussi dans les logiques de mobilités géographiques et sociales des acteurs (accès à telle ou telle connaissance, aide et conseil personnalisé, appartenance au tissu associatif...). Nous notons, par exemple, que dans le Sud-Aveyron, les réseaux associatifs sont un levier pour l'innovation mais également un lieu de partage pour des personnes parfois excentrées ou marginalisées.
L'innovation se construit ainsi dans l'interaction, autour d'individus qui se côtoient et qui ont des réseaux communs. La notion de réseaux comme "ensemble particulier d'interrelations entre un ensemble limité de personnes, avec la propriété supplémentaire que les caractéristiques de ces interrelations sont considérées comme une totalité" (cf. Clyde Mitchell ${ }^{(4)}$, cité dans Gaglio, 20II), peut être utilisée pour interpréter le comportement social des personnes impliquées dans le processus d'innovation patrimoniale alimentaire. Dans ce cas, l'innovation se diffuse de proche en proche, comme une toile d'araignée, se tissant progressivement.

L'appartenance au réseau joue le rôle d'accompagnant ou de facilitateur d'innovation. L'exemple du réseau des amicales des Aveyronnais à Paris nous montre l'influence que peut avoir une telle appartenance dans la diffusion et la reconnaissance du processus d'innovation agroalimentaire. L'amicalisme aveyronnais, par sa structuration et sa force d'adhésion, contribue fortement au développement et au rayonnement du patrimoine alimentaire nord-aveyronnais. L'aligot, le fromage de Laguiole, la fouace ou la charcuterie de l'Aubrac doivent en partie leur renommée et leur qualification au réseau amicaliste parisien soucieux de promouvoir et de diffuser une culture alimentaire à l'extérieur des frontières aveyronnaises. Ambassadeurs, ou facilitateurs, ces réseaux contribuent à l'adoption et à la diffusion du processus d'innovation. 
Nous repérons également la présence et l'activation de réseaux ou partenariats plus "formels" : des partenariats techniques, technologiques (Direction des services vétérinaires), de qualification (Institut régional pour la qualité alimentaire en Midi-Pyrénées), de contrôle (Qualisud), de conseils et d'appui aux projets (comité d'expansion, conseil général) qui cherchent de plus en plus souvent à accompagner les processus d'innovation et à s'en faire le relais. L'intervention de ces réseaux formels constitue une institutionnalisation des pratiques innovantes, favorisant leur appropriation. La constitution d'un réseau de professionnels, par exemple autour de produits, témoigne d'une volonté d'ouverture, de rayonnement et de recherche de personnes ressources, capables de soutenir la dynamique d'innovation (chambres consulaires, partenaires publics et privés). Les réseaux ou partenariats formels les plus connus ou reconnus se comptent parmi les filières de produits agroalimentaires sous signe officiel de qualité (IGP, AOP, label rouge). Les réseaux ou partenariats de prestataires touristiques (Bienvenue à la ferme, Accueil paysan, De ferme en ferme), les réseaux ou partenariats agricoles (Cuma coopératives d'utilisation de matériel agricole, syndicats agricoles...) ou ceux du développement local (parcs naturels régionaux, chambres de commerce et d'industrie) sont également largement cités. L'innovation se construit d'appuis institutionnalisés ou légitimés, comme le conseil ou l'aide technique d'acteurs reconnus dans la société locale (PNR, CCI, chambres d'agriculture, etc.).

Enfin, au-delà du réseau, nous voyons émerger la présence d'acteurs relais, "leaders" qui, par leur vision du territoire, par leur "esprit d'innovation" ou leur motivation à innover font aboutir les démarches d'innovation. Olivier de Sardan (1995) souligne le rôle prépondérant de ces porteurs sociaux, ou facilitateurs d'innovation, qui, par leur légitimité ou crédibilité sociale, contribuent grandement à la diffusion de l'innovation. Ces acteurs influents, "passeurs" d'innovation, peuvent appartenir aux institutions locales (organismes agricoles, structures de développement), inspirent confiance et aident à la décision sur des questions propres au territoire. Ils peuvent également appartenir à la société civile locale, dans la frange la plus en contact avec l'extérieur (élites, anciens migrants, entrepreneurs, militants associatifs, notables). Ces acteurs, assimilables à la figure du "marginal sécant" de Crozier et Friedberg (1977), constituent des courroies de transmission entre le monde extérieur et le territoire. Le processus d'innovation dépend alors de leur présence ou de leur influence sur la scène locale. Porteurs d'une assise socio-économique ou politique, ces acteurs se révèlent, par leur statut ou leur légitimité, comme des "passeurs" ou des médiateurs incontournables de l'innovation. En Aubrac par exemple, André Valadier, élu local et régional, res- ponsable du comité des produits laitiers à l'Inao (Institut national des appellations d'origine) pendant de nombreuses années, a joué auprès de la coopérative Jeune Montagne notamment, mais aussi auprès d'autres acteurs locaux du monde agricole et socio-économique, le rôle de leader, de révélateur, de "conscientiseur". Il a été également à l'initiative des démarches de structuration des filières viande d'Aubrac : Bœuf fermier Aubrac et Fleur d'Aubrac, qui sont tout autant de formes d'innovation organisationnelle pour les éleveurs et bouchers du territoire.

L'innovation patrimoniale alimentaire prend sa source dans des logiques de création, d'invention ; ces dernières sont elles-mêmes conçues par une volonté de créer, par des dynamiques de rupture, de mobilités tant personnelles que professionnelles ou touristiques. Toutefois, l'innovation ne saurait être assimilée à ce seul acte créateur. L'innovation patrimoniale, au-delà de la nouveauté, procède de l'appropriation et de l'incorporation de la pratique par un ensemble social. S'appuyant sur l'ancrage spatial et temporel des productions alimentaires mais également sur l'activation de réseaux et l'intervention d' "acteurs leaders", le processus d'innovation est pluriel. Il se construit de manière variable selon les types de structures et d'acteurs, selon les territoires et les patrimoines alimentaires valorisés, engendrant confrontations et divergences. 
CONFRONTATIONS

ET DIVERGENCES AUTOUR

DE L'INNOVATION

PATRIMONIALE ALIMENTAIRE

Le processus d'innovation patrimoniale alimentaire n'est ni linéaire, ni systématique. Il est le lieu de ruptures, de conflits, de remise en cause des régulations antérieures et des certitudes. Son appropriation est traversée d'obstacles et de freins, donnant à voir des représentations contradictoires, notamment dans le rapport à la tradition et à l'héritage. La réflexion qui suit s'interroge sur la manière dont l'innovation interpénètre (ou pas) la patrimonialisation, à travers les pratiques et les représentations de la valorisation. Elle présente, sous la forme de processus, les articulations observées entre innovation et patrimonialisation alimentaire en s'appuyant sur le discours des acteurs interrogés et sur la manière dont ils se représentent et vivent les dynamiques à l'œuvre. Différentes grilles de lecture ou cadres de référence se mêlent, se croisent et se confrontent, constituant des repères ou déterminants contrastés de l'innovation patrimoniale. Aussi, si l'identification du patrimoine, à l'instar du débat sur la construction des identités locales, recouvre le plus souvent une dimension unitaire ou unificatrice, elle peut aussi être source de division et de désunion sociale. Le patrimoine alimentaire et l'innovation qui y est associée apparaissent comme un enjeu de représentations contradictoires, engendrant des relations par- fois conflictuelles entre les acteurs. Les trois territoires de notre étude font en effet apparaître des divergences dans l'interprétation et le sens donné à l'articulation patrimoine alimentaire / innovation. Cette articulation est analysée ici à travers une typologie de processus qui rend compte des liens et du rapport existant entre continuité et changement, entre tradition et modernité. La typologie présentée repose sur l'analyse des représentations des acteurs et des liens qu'ils entretiennent avec la dimension traditionnelle et innovatrice des patrimoines alimentaires.

\section{Quand la tradition \\ alimentaire supplante l'innovation}

Ce processus met en avant la tradition alimentaire comme dominante dans l'action de valorisation patrimoniale. Il se caractérise par des pratiques et des représentations centrées sur une vision traditionnelle des produits et de l'héritage alimentaire, sur leur continuité et leur permanence. Dans ce scénario, la tradition supplante largement l'innovation jusqu'à l'ignorer ou l'écarter. Nous observons ici une logique de valorisation centrée sur la transmission de l'héritage, du passé et sur la reproduction d'éléments hérités. La patrimonialisation demeure, dans ce scénario, assimilée au fait traditionnel.

Ce mode d'interprétation patrimoniale que nous qualifions de "conservateur" ou de "traditionaliste" se rencontre sur nos trois territoires d'étude. Soutenu par des acteurs "gardiens du temple" ou passeurs de mémoire, il met l'accent sur la continuité entre le passé et le présent, la perpétuation et la constance de l'héritage. Nous retrouvons ici les analyses de Corbeau autour du "métissage refusé", du regard "muséifiant" sur les cultures alimentaires, contestant le changement (Corbeau et Poulain, 2002). Les productions alimentaires locales apparaissent comme des héritages à conserver, à maintenir et à transmettre en l'état. La mémoire et l'histoire deviennent alors des chevaux de bataille, des espaces de sauvegarde absolus, "intouchables", sacralisés, à l'image de certains secrets de famille et traditions culinaires locales. "Cette boutique est toujours restée dans la tradition familiale. Ma mère, ma sour et moi-même faisons toujours de la même manière, depuis 1911 nous n'avons rien changé, ni la recette..., nous avons notre secret" (boulanger, Gal Aubrac, Olt et Causses, Nord-Aveyron).

Aussi, parfois décriés pour leur immobilisme et une certaine idéologie conservatrice, ces acteurs s'opposent à l'apport des techniques, au renouvellement des savoirs, à toute création. "Je me suis bien gardé d'innover, je ne changerai pas. Je n'ai pas changé, je n'ai pas agrandi. Je reste ce que je suis et je garde ce que l'on m'a transmis" (boulanger, Gal Aubrac, Olt et Causses, NordAveyron). L'innovation est ici perçue comme une atteinte au produit local, une trahison d'un héritage, une infidélité faite au passé, une voie vers l'uniformisation des produits et des pratiques alimentaires. 
La dimension "traditionnelle" omniprésente dans le discours de certains acteurs est une garantie de sens et de transparence pour le mangeur. L'ancrage spatial et temporel des produits doit être sauvegardé au risque de dénaturer et de trahir l' "authenticité" tant recherchée par les consommateurs. Avec fierté et revendication, la tradition et la transmission ici alimentaires sont affichées comme salvatrices d'une identité locale en lutte contre toutes formes d'uniformisation et de perte du goût. "Je lutte contre la perte et la mondialisation du goût par le maintien d'une véritable cuisine, originelle, comme les anciens nous l'ont appris ; ça c'est du patrimoine" (restaurateur, Gal Pays d'Armagnac, Gers). Nous relevons dans les discours et les dépliants publicitaires d'innombrables termes évocateurs associés au passé et à la continuité : le "rustique", 1" authentique", le "naturel", le "comme à l'ancienne" ou le "comme autrefois" ou le "fait maison" scandent bon nombre de propos rejetant radicalement tout dispositif de création ou d'invention, jugé dangereux et destructeur.

De nombreux exemples de valorisation rencontrés chez des restaurateurs, pâtissiers, charcutiers, etc. démontrent des techniques inchangées issues du secret et du tour de main. Des techniques de valorisation fondées sur le respect de certaines règles de fabrication, sur la sauvegarde optimale d'une mémoire familiale, sur un minimum d'apports extérieurs (invention réduite de recettes, présence minimale de machines dans les ateliers de fabrication, main d'œuvre familiale abondante préservant l'élaboration manuelle des produits, etc.) traduisent chez certains acteurs une volonté de mobiliser l'héritage alimentaire comme une arme de survie et de maintien socio-économique. "Faire de la qualité", c'est alors veiller à la transmission, au respect de l'héritage, et repousser l'arrivée trop brutale d'emprunts et d'influences culinaires ou agro-alimentaires extérieurs.

Cette vision du patrimoine alimentaire et de sa valorisation s'avère à l'antipode de la mondialisation alimentaire. Contestée par certains, elle est alors perçue comme une forme réactionnaire de représentations trop passéistes, cristallisant et paralysant le sens donné au patrimoine alimentaire.

\section{Quand la création alimentaire s'éloigne de la tradition}

Une deuxième approche, à dominante "innovatrice" ou "créatrice", se caractérise par l'évocation dominante du lien à la modernité. Contrairement à l'analyse précédemment évoquée, le patrimoine alimentaire est ici un support d'invention et d'évolution ; projetée dans le futur, l'interprétation du patrimoine s'inscrit en opposition à l'approche conservatrice, de maintien et de sauvegarde, jugée trop archaïque et trop statique. Nous repérons là des discours centrés sur l'innovation et la création permanente, où sont définis des processus d'action et de valorisation dynamiques et évolutifs, caractérisés par des changements et du renouvellement perpétuels. Cette approche rejoint l'analyse de Joseph Schumpeter selon laquelle l'innovation est fondamentalement une "destruction créatrice", qui transforme, fabrique du neuf en même temps qu'elle démolit de l'ancien (Schumpeter, 1951, cité dans Gaglio, 20 I I). L'inconnu, l'inédit, l'improvisé, la nouveauté s'avèrent fédérateurs, qualifiant la fonction d'adaptation du patrimoine. L'arrivée et l'utilisation de nouveaux ingrédients, inconnus jusqu'alors, la mise en place de nouveaux modes de cuisson, la création et l'invention de connaissances comme l'apport de nouvelles techniques s'accompagnent d'un rejet de l'ancien, du "déjà fait" et de l'ordre établi. À l'opposé d'une vision traditionnelle du terroir et de ses produits, ce mode d'interprétation du patrimoine permet d'accéder à un renouvellement des savoirs et savoir-faire, conduisant à une définition non statique et sans cesse renouvelée de l'héritage alimentaire, ici intégré à l'histoire actuelle. "Mon patrimoine, c'est ce que j'invente tous les jours en fonction des émotions ressenties, j'assemble des éléments, des produits nouveaux. Mon patrimoine n'est pas celui de mes grands-mères, ce n'est surtout pas le jambon et la nappe à carreaux!” (restaurateur, Gal Aubrac, Olt et Causses, Nord-Aveyron).

L'innovation est au centre du processus de valorisation, s'éloignant, diluant et étouffant le plus souvent 
la dimension spatiale et temporelle du patrimoine alimentaire. Les innovations technologiques (introduction de nouvelles machines, changements des processus de fabrication, etc.) apparaissent centrales dans l'activité de valorisation, comme garantes de la prospérité et de la survie du patrimoine alimentaire. L'efficience et la productivité du produit priment sur la sauvegarde et la conservation. Dans l'industrie du roquefort, par exemple, on observe un renouvellement et une croissance des innovations technologiques notamment, dans le mode de fabrication et d'affinage du fromage (arrivée de machines, industrialisation du processus de fabrication). Les notions de productivité, de marketing, de politiques d'investissements, de direction d'exploitation, de processus structurent les discours des acteurs rencontrés, soulignant l'intégration du patrimoine alimentaire dans un système avant tout économique et productif. La progression technicoéconomique du produit marque l'évolution du patrimoine alimentaire, qui n'en finit pas de se renouveler et de se réinventer.

En outre, si les dimensions traditionnelles s'estompent dans les pratiques de valorisation observées, elles se retrouvent rehaussées, voire exacerbées dans les énoncés promotionnels. Nous constatons alors, à l'opposé des pratiques, une mystification et une construction d'images autour du passé et de l'héritage alimentaire ; la tradition et ses signes sont ici utilisés comme des outils de communication, de médiatisation afin de rassurer et de séduire le consommateur. Cette "tradition communiquée”, réduite à l'image marketing, s'oppose sensiblement à une "tradition vérité" ou vivante, rencontrée par ailleurs. La dimension patrimoniale, notamment à travers son image, peut servir de point d'appui ou de levier à l'innovation et à l'invention de nouveaux produits. La tradition, le patrimoine, l'ancrage au lieu ou au temps apparaissent comme des supports de communication ou de mise en image.

\section{Quand l'innovation réinvente l'héritage alimentaire}

À la croisée des deux approches précédentes, un dernier type de discours et de processus s'apparente à une idéologie de l'adaptation, prônant la reproduction tout comme la création, le respect de la mémoire ainsi que le changement. "Innovateur" et "traditionnel" à la fois, il renvoie à des pratiques hybrides et syncrétiques, définissant la tradition comme une construction sociale en perpétuel mouvement. Nous rejoignons ici l'analyse de Lenclud: "Loin d'être une 'vérité" qui s'impose aux hommes d'aujourd'hui, la tradition est d'abord un 'point de vue', une interprétation qu'ils développent sur ce qui les a précédés. Elle sélectionne les éléments $d u$ passé, elle les inscrit dans un ordre différent et leur confère un sens pour le présent, suivant des modes de transmission des héritages et de la mémoire socialement déterminés. La tradition est avant tout un message culturel" (Lenclud, 1987).
Dans cette approche, le patrimoine alimentaire se situe dans un va-et-vient de représentations inspirées du passé, du présent et du futur. La mémoire culinaire ou alimentaire se trouve réutilisée, réactualisée, et réinjectée dans le présent. La patrimonialisation est envisagée comme la réinvention d'un héritage à recomposer et à renouveler. À la différence des discours dits "innovateurs" qui s'éloignent d'une mémoire prescrite, l'approche mixte s'y réfère, se l'approprie, pour participer à sa régénérescence. "Le patrimoine, c'est une succession d'histoires jusqu'à aujourd'hui; c'est une évolution constante à laquelle nous devons participer en tenant compte de ce qui vient de se passer avant" (animatrice de Pays, Gal Pays d'Armagnac, Gers).

Fondé sur la complémentarité plus que sur l'antinomie, ce scénario plutôt "consensuel” manie avec justesse tradition et modernité alimentaire. La pratique de la "pluri-compétence" (articulation de compétences commerciales, relationnelles, d'organisation, etc.), l'intégration de réseaux de coopération et d'action, l'ajout et l'emprunt de savoirs et savoir-faire extérieurs caractérisent un processus de valorisation marqué par le métissage et la construction. Nous observons sur les territoires enquêtés un foisonnement d'initiatives locales rassemblées autour de réseaux de qualité, d'offres agroalimentaires spécifiques et différenciées, combinant par exemple sur une même entité spatiale les segments amont, centre et aval de la chaîne 
de valorisation alimentaire. Ce processus dynamique vise à construire et à inventer de nouvelles formes de valorisation axées sur les ressources territoriales mais ouvertes à la nouveauté. L'innovation est ici à dominante sociale et organisationnelle et a pour fonction la réinvention d'un "faire ensemble", d'un nouveau rapport au territoire et à ses ressources patrimoniales. Ces dernières sont perçues comme des leviers de renouveau socio-économique au service de l'avenir des territoires : [...] L'innovation, c'est la façon de le travailler [le produit] ou d'améliorer la qualité de quelque chose. Moi, je ne vais pas chercher des produits ailleurs. Je fais une glace an fromage de Laguiole que je sers avec des truites et des farçous. Ce sont des produits qui sont typiques d'ici mais que l'on fait différemment" (restauratrice, Gal Aubrac, Olt et Causses, Nord-Aveyron).

Ce processus de patrimonialisation s'appuie sur une combinaison de deux sources complémentaires : d'une part, la connaissance et les usages du produit, issus de la mémoire enracinée dans l'histoire des groupes sociaux, et, d'autre part, les divers apports, emprunts externes issus d'une connaissance technique ou de la conception de nouvelles épreuves. Si le changement et l'invention peuvent tendre à une banalisation du produit, en le normalisant et en inhibant sa dimension patrimoniale, ils apparaissent aussi $a$ contrario comme des enrichissements complémentaires, renforçant la spécificité et la typicité du produit.
"L'emprunt d'éléments exogènes ne saurait se résumer à un simple implant sans relation à la mémoire. [...] La greffe ou l'hybridation semblent des métaphores plus conformes à l'esprit de l'activité combinatoire dans la mesure où l'emprunt ne réduit pas la part des éléments préexistants, il s'y associe de façon novatrice" (Casabianca et Sainte-Marie, 1998). Articuler judicieusement héritage et innovation, permanence et mouvement, reproduction et création contribue à produire une nouvelle connaissance sociale. Le patrimoine alimentaire et sa valorisation participent alors de la production d'identité et d'unité.

La notion "d'héritage vivant" au service du futur annonce un modèle novateur de développement patrimonial, ici alimentaire. Associé souvent au développement touristique ou aux stratégies de mobilités, ce processus se caractérise par l'intégration constante du changement, que ce soit dans l'invention de nouvelles formes de valorisation (mise en place de marchés de producteurs de pays, création de nouvelles organisations) ou dans l'évolution progressive de produits locaux (proposition de nouveaux plats ou recettes). Le tourisme et l'arrivée de nouvelles populations sur les territoires sont vécus comme des moteurs de la construction de ce modèle. Le touriste citadin devient militant de ce qu'il a vu et compris en vacances. Il est à la fois force de réappropriation patrimoniale pour les populations locales en participant à la différenciation et à la légitimation sociale des productions alimentaires, mais également facteur d'invention et de renouvellement. "J'appelle le tourisme avec insistance parce que je sais ce qu'il nous apporte, je sais ce qu'il pourrait apporter et je sais ce que l'on deviendrait s'il n'était plus là. Difficile avec les agriculteurs : pour eux les touristes encombrent, nous cassent les pieds. Plus de restaurants, plus d'hôtels : ils apportent le développement du chemin de Saint-Jacques par exemple. Le touriste est un consommateur potentiel, il réagit en fonction de ses besoins mais aussi de ses désirs. Quand je vois que ces touristes reçoivent des réponses fondées, je les vois devenir militants" (un élu, ancien responsable de coopérative, Gal Aubrac, Olt et Causses, Nord-Aveyron).

Le touriste, porteur d'une reconnaissance extérieure participe à la recomposition du produit de terroir, le confrontant à des valeurs externes, liées à la construction actuelle d'un imaginaire touristique et gastronomique. Au lieu de s'opposer et de s'exclure mutuellement, tradition et modernité semblent non seulement coexister mais se renforcer réciproquement dans un même processus de valorisation. Le phénomène touristique, considéré comme composante des sociétés modernes, influe sur ce processus combinatoire. Il s'avère ainsi vecteur de changement, de recomposition patrimoniale mêlant le nouveau à l'ancien, participant à l'incorporation de la tradition à une nouvelle société rurale en mutation. "Vous êtes obligés de vous adapter au milieu, le milieu est 
actif, il est acteur, vous me permettrez de dire qu'il est réactivé et il est de ce fait un allié permanent. Lorsque vous êtes dans un espace territorial avec ces spécificités, ce milieu donne des signaux et peut vous rappeler à l'ordre. Remettre des pas dans ces traces sans qu'il ne soit fait obligation de porter des sabots de bois. C'est peut-être pour ne pas avoir à porter ces sabots de bois qu'il y a lieu d'innover le matériel dont on a parlé tout à l'heure, pour rester dans une logique produit. Si mes petits-enfants restent sur le territoire, ils seront obligés de composer avec ce territoire. Il y a une espèce de synergie" (un élu, responsable de coopérative, Gal Aubrac, Olt et Causses, Nord-Aveyron).

Le patrimoine alimentaire, vivant au quotidien, subit des modifications en même temps qu'il s'enrichit, se déploie et donne naissance à de nouvelles formes d'inventions culinaires ou alimentaires. Porté par des mécanismes de transmission auxquels s'ajoutent des emprunts ou apports exogènes (technicités nouvelles liées à l'hygiène, invention de recettes, intégration à de nouveaux réseaux de distribution, etc.), il intègre un processus naturel d'évolution locale.

Ce processus "hybride" de patrimonialisation se retrouve, plus ou moins consciemment, dans quelques discours d'artisans, de commerçants, de chefs d'entreprise pour qui passé et avenir sont mêlés au point de ne former plus qu'un. "On n'invente jamais rien, on le modifie, on le fait à sa sauce. Quant on arrive dans un pays, on s'appuie sur l'existant, les savoir-faire en place" (producteur de miel, Gal AMIE, Sud-Aveyron). Au-delà d'une conviction forte de fonctionner comme autrefois, imprégnés d'un savoir et savoir-faire gastronomiques, les producteurs occultent parfois les modes d'adaptation constants opérés au quotidien (intégration au réseau Bienvenue à la ferme, à diverses associations, participation aux formations, application des normes européennes, etc.). Peu conscients de leur transformation, les producteurs, plus généralement les acteurs de la valorisation rencontrés, "cultivent un authentique”, entretiennent et développent un existant patrimonial, avançant dans une modernité dont ils ont parfois peu conscience.

\section{CONCLUSION}

Processus éminemment social, l'innovation patrimoniale alimentaire renvoie à des logiques de ruptures, de mobilités tant personnelles que professionnelles ou touristiques. Portée par la création ou l'invention personnelle, elle est également le résultat d'une adhésion collective et de l'appropriation de nouvelles pratiques par un groupe social. Ce processus d'appropriation locale est conditionné par l'inscription temporelle et spatiale de la nouveauté en même temps qu'il s'appuie sur l'activation de réseaux et l'intervention d' "acteurs leaders" ou "agents passeurs", investis et légitimes aux yeux du groupe local. Nous l'avons vu dans cet article : l'innovation dans le champ des patrimoines ali- mentaires relève de mécanismes de transmission, de co-construction sociale, d'influences diverses, d'emprunts extérieurs, de croisements de pratiques de valorisation, de processus combinatoires complexes. Autant de mécanismes qui nourrissent les processus sociaux de développement local, et accompagnent les dynamiques de projets. L'innovation patrimoniale ne va pas de soi ; elle est traversée d'obstacles et de contradictions dans les représentations et les pratiques des acteurs, notamment dans son rapport à la tradition et à la modernité alimentaire. Cohabitent ou se confrontent, selon les cas, différents types de représentations et de conceptions liées à l'identification du patrimoine alimentaire et aux stratégies d'innovation. Ou totalement "traditionnels", refusant l'innovation, ou radicalement "innovateurs", oubliant la tradition, ou bien encore "mixtes" ou "métissés", renouvelant au quotidien l'héritage alimentaire, les modes d'interprétation du patrimoine alimentaire, et avec lui de ses stratégies de valorisation, divisent et écartent toute vision unitaire et consensuelle de ce patrimoine à l'échelle locale. 
NOTES

(I) Cette contribution s'appuie sur deux publications sources : Jacinthe BESSIÈRE (dir.), Innovation et patrimoine alimentaire en espace rural, coll. "Update Sciences \& technologies", Éditions Quae, 2012 ; Jacinthe BESSIÈrE et Laurence TiBère, "Innovation et patrimonialisation alimentaire : quels rapports à la tradition ?", Ocha, Observatoire Cniel des habitudes alimentaires, mars 2010 . En ligne à partir de [http://wnw.lemangeurocha.com/fileadmin/im ages/sciences_humaines/Texte_exclusif_BESSIERE_et_TIBERE_innovation_et_patrimonialisation.pdf].

(2) Ces trois territoires ont été intégrés au programme Leader, visant à soutenir le développement rural européen ; le programme Leader+ a eu pour spécificité de mettre en avant des thématiques considérées comme des moteurs d'innovation pour les territoires ruraux. L'une d'entre elles fut centrée sur "la valorisation des produits locaux".

(3) Une trentaine d'entretiens semi-directifs ont été réalisés sur chacun des territoires d'étude, auprès de deux principales catégories d'acteurs : les acteurs socioprofessionnels impliqués dans les stratégies de valorisation patrimoniale alimentaire (restaurateurs, directeurs de coopératives, d'entreprises agro-alimentaires, etc.), les acteurs du développement territorial (élus locaux, responsables d'associations, offices de tourisme, etc.). Les premiers ont été interrogés sur leur parcours individuel et professionnel, sur leur perception de l'innovation et sur leur manière de la mettre en œuvre. Les seconds devaient également s'exprimer sur leur vision de l'innovation, en particulier en relation avec leur territoire.

(4) Clyde MiTCHELL, Social networks in urban situations, Manchester University Press, 1969.

\section{RÉFÉRENCES BIBLIOGRAPHIQUES}

Norbert ALTER, L'Innovation ordinaire, coll. "Sociologies", Puf, 2000.

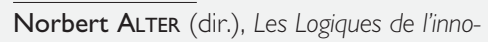
vation. Approche pluridisciplinaire,

La Découverte, 2002.

Jacinthe BESSIÈRE, Valorisation du patrimoine gastronomique et dynamiques de développement territorial. Le haut plateau de l'Aubrac, le pays de Roquefort et le Périgord noir, coll. “Logiques sociales”, L'Harmattan, 2001.

Jacinthe BESSIĖRE, "Les terroirs de valorisation gastronomique", dans Claire DelFOSSE (dir.), La Mode du terroir et les produits alimentaires, La Boutique de l'histoire, 20I I.

Jacinthe BESSIÈRE (dir.), Innovation et patrimoine alimentaire en espace rural, coll. "Update Sciences \& technologies", Éditions Quae, 2012.

Jacinthe BESSIÈrE, '“Héritagisation', a challenge for tourism promotion and regional development: example of food heritage", Joumal of Heritage Tourism, Routledge, Taylor \& Francis Group, mis en ligne en 2013.

[http://dx.doi.org/10.1080/1743873X.2013. $770861]$.

Jacinthe BESSIÈRE et Laurence TIBÈRE,

"Innovation et patrimonialisation alimentaire : quels rapports à la tradition ? enquête dans trois territoires ruraux de Midi-Pyrénées", Ocha, Observatoire Cniel des habitudes alimentaires, mis en ligne le 8 mars 2010. À partir de : http://www.lemangeur-

ocha.com/fileadmin/images/sciences_humai nes/Texte_exclusif_BESSIERE_et_TIBERE_ innovation_et_patrimonialisation.pdf [consulté le ler septembre 2012]. 
$\overline{\text { Jacinthe BESSIÈrE }}$ et Laurence TIBÈrE,

"Patrimoines alimentaires", Anthropology of Food, $n^{\circ} 8,2011$. En ligne à partir de [http://aof.revues.org/index6782.html]. François CASABIANCA et Christine DE SAINTE-MARIE, "Concevoir des innovations pour les produits typiques", dans INRASAD/CIRAD-TERA/CnEARC, Atelier Dynamiques territoriales d'innovation, modalités sociales, combinaisons et confrontations de savoirs, Inra, 1998.

Jean-Pierre CORBEAU et Jean-Pierre

PoulaIN, Penser l'alimentation. Entre imaginaire et rationalité, Privat, 2002.

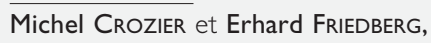

L'Acteur et le système. Les contraintes de l'action collective, coll. "Sociologie politique", Seuil, 1977.

Olivier ETCHEVERRIA, "Les tensions fondatrices du développement local autour du restaurant Bras à Laguiole : 'centralité', qualité et créativité", Norois, $n^{\circ} 219$ (numéro spécial : "Le restaurant, outil de développement local ?'), Presses universitaires de Rennes, septembre 201 I.

Marie-Blanche FourCADE et Caroline

LEGRAND, Patrimoines des migrations,

migrations des patrimoines, coll.

"Intercultures", Presses de l'université Laval, 2008.

Gérald GAGLIO, Sociologie de l'innovation, coll. "Que sais-je ?", Puf, 20। I.

Gérard LENCLUD, “La tradition n'est plus ce qu'elle était", Terrain, n 9, 1987//23.

Jean-Pierre OLIVIER DE SARDAN,

Anthropologie et développement. Essai en socio-anthropologie du changement social, coll. "Hommes et Sociétés", Karthala, 1995.
Jean-Pierre PoulAIN, "Le goût du terroir et le tourisme vert à l'heure de l'Europe", Ethnologie française, tome XXVII, 1997.

Joseph SCHUMPETER, Capitalisme, socialisme et démocratie, Payot, 195 I (I parution 1942). 